\title{
Oblique water-wave scattering by small undulation on a porous sea-bed
}

\author{
S.C. Martha ${ }^{\text {a }}$, S.N. Bora ${ }^{a}$, A. Chakrabarti ${ }^{b, *}$ \\ ${ }^{a}$ Department of Mathematics, Indian Institute of Technology Guwahati, Guwahati 781039, India \\ ${ }^{\mathrm{b}}$ Department of Mathematics, Indian Institute of Science, Bangalore 560012, India
}

Received 25 August 2006; received in revised form 22 July 2007; accepted 26 July 2007

Available online 4 September 2007

\begin{abstract}
The problem of scattering of obliquely incident surface water waves by small undulation on a sea-bed is considered for solution by assuming that the bed is composed of porous material of a specific type. The reflection and transmission coefficients are determined approximately after assuming a perturbation analysis in conjunction with the Fourier transform technique, in terms of the first order of smallness of an undulation parameter $\varepsilon$, introduced in the description of the sea-bed. The special case of a patch of sinusoidal ripples on the bed is handled in detail and numerical results are presented graphically.
\end{abstract}

(c) 2007 Elsevier Ltd. All rights reserved.

Keywords: Scattering; Reflection and transmission coefficients; Linear water-wave theory; Perturbation technique; Porous sea-bed

\section{Introduction}

The problems of interaction of surface waves with a preexisting (fixed) pattern of undulation on an otherwise flat bed are important for their possible applications in the areas of coastal and marine engineering. In practical coastal engineering, porosity of the bed also becomes an exceedingly important aspect to be handled. Miles [9] and Davies and Heathershaw [3] considered the problem of water-wave scattering by a sea-bed with an undulating bottom topography. Mandal and Basu [5] generalized the problem of Miles [9] to include the effect of surface tension at the free surface. Martha and Bora [6] solved the problem of oblique water-wave diffraction by small undulation on the sea-bed obtaining detailed results for a patch of sinusoidal ripples, as a specific example.

The above works focused only on the wave motion of the free water region and the effect of porosity of the sea-bed was not taken into account. Mase and Takeba [7], Zhu [11] and Silva et al. [10] considered water-wave reflection and/or transmission problems where a porous medium was assumed to lie on a sea-bed of varying quiescent depth.

In the present note we have considered a scattering problem involving a porous bed profile, along with undulation.

\footnotetext{
* Corresponding author. Tel.: +91 802293 2571; fax: +91 8023600416

E-mail address: alok@math.iisc.ernet.in (A. Chakrabarti).
}

We formulate the problem for the case of an obliquely incident train of surface waves. The motion of the fluid inside the porous bed is not analysed here and it is assumed that the fluid motions are such that the resulting boundary condition on the sea-bed as is used in this note, holds good and depends on a known parameter $G^{\prime}$, called the porosity parameter. In this case, the governing boundary value problem (BVP) is reduced to a simpler BVP for the first order correction of the potential using perturbation analysis which involves a small parameter $\varepsilon$ being present in the representation of the small undulation of the porous sea-bed. Then the solution of the BVP is obtained by using the Fourier transform technique and the reflection and transmission coefficients are obtained approximately. For a special form of the undulation as represented by a patch of sinusoidal ripples, numerical results for these coefficients are obtained which are presented graphically.

It may be remarked that the understanding of the effect of sinusoidal variations of the sea-bed helps in analysing more general forms of smooth undulated sea-beds by using standard Fourier analysis.

\section{Formulation of the problem}

Using right-handed rectangular Cartesian coordinates $(x, y, z)$ with the $x z$-plane representing the undisturbed free 
surface of the sea, the $y$-axis being measured positive vertically downwards and also assuming linear water-wave theory and irrotational motion of water in the sea, we wish to solve the following BVP for the complex-valued potential function $\psi(x, y, z)$ :

$$
\begin{aligned}
& \frac{\partial^{2} \psi}{\partial x^{2}}+\frac{\partial^{2} \psi}{\partial y^{2}}+\frac{\partial^{2} \psi}{\partial z^{2}}=0, \quad \text { in } 0 \leq y \leq h+\varepsilon c(x), \\
& -\infty<x, z<\infty \\
& \frac{\partial \psi}{\partial y}+K \psi=0, \quad \text { on } y=0,-\infty<x, z<\infty \\
& \frac{\partial \psi}{\partial n}-G^{\prime} \psi=0, \quad \text { on } y=h+\varepsilon c(x),-\infty<x, z<\infty
\end{aligned}
$$

where $y=h+\varepsilon c(x)$ represents the undulating porous sea-bed with $\varepsilon$ as a non-dimensional parameter and $c(x)$ is a continuous bounded function describing the shape of the bottom, $c(x) \rightarrow$ 0 as $|x| \rightarrow \infty$. In Eqs. (1)-(3), $K=\sigma^{2} / g, \sigma$ is the angular frequency of the incoming water-wave train with time dependence $\mathrm{e}^{-\mathrm{i} \sigma t}$, dropped throughout the analysis, $g$ is the acceleration due to gravity, $\partial / \partial n$ denotes the normal derivative at a point $(x, y, z)$ on the bottom, and $G^{\prime}$ is the porous effect parameter corresponding to the sea-bed under consideration.

We assume that an obliquely incident progressive wave train as given by the function

$\psi_{0}(x, y, z)=\phi_{0}(x, y) \mathrm{e}^{\mathrm{i} v z}$,

where

$\phi_{0}(x, y)=\frac{K \sinh k_{0} y-k_{0} \cosh k_{0} y}{K \sinh k_{0} h-k_{0} \cosh k_{0} h} \mathrm{e}^{\mathrm{i} \mu x}$

with $\mu=k_{0} \cos \theta, v=k_{0} \sin \theta(0 \leq \theta<\pi / 2), \theta$ being the angle of incidence and the wave number $k_{0}$ being the unique positive real root of the equation

$K+G^{\prime}=\left(k+G^{\prime} K / k\right) \tanh k h$,

coming from the direction of negative infinity, gets partially reflected by and partially transmitted over the bottom undulation.

Then the problem is the determination of the function $\psi(x, y, z)$ satisfying the relations (1)-(3) and the following farfield condition:

$\psi(x, y, z) \sim \begin{cases}\psi_{0}(x, y, z)+R \psi_{0}(-x, y, z), & \text { as } x \rightarrow-\infty \\ T \psi_{0}(x, y, z), & \text { as } x \rightarrow+\infty\end{cases}$

where $R$ and $T$ denote, respectively, the reflection and transmission coefficients which are also to be determined in the course of the solution of the problem.

\section{Solution procedure}

The bottom condition (3) can be approximated up to the first order of the small parameter $\varepsilon$ as

$$
\frac{\partial \psi}{\partial y}-\varepsilon\left[\frac{\partial}{\partial x}\left\{c(x) \frac{\partial \psi}{\partial x}\right\}+c(x) \frac{\partial^{2} \psi}{\partial z^{2}}\right]
$$

$$
-G^{\prime}\left[\psi+\varepsilon c(x) \frac{\partial \psi}{\partial y}\right]=0 \quad \text { on } y=h .
$$

We write in view of the geometry of the problem, as well as the form of the incident wave,

$\psi(x, y, z)=\phi(x, y) \mathrm{e}^{\mathrm{i} v z}$.

Then $\phi(x, y)$ is the solution of the following BVP:

$$
\begin{aligned}
& \left(\nabla^{2}-v^{2}\right) \phi=0, \quad \text { in }-\infty<x<\infty, 0 \leq y \leq h \\
& \frac{\partial \phi}{\partial y}+K \phi=0, \quad \text { on } y=0 \\
& \frac{\partial \phi}{\partial y}-\varepsilon\left[\frac{\partial}{\partial x}\left\{c(x) \frac{\partial \phi}{\partial x}\right\}-v^{2} c(x) \phi(x, y)\right] \\
& -G^{\prime}\left[\phi+\varepsilon c(x) \frac{\partial \phi}{\partial y}\right]=0, \quad \text { on } y=h \\
& \phi(x, y) \sim \begin{cases}\phi_{0}(x, y)+R \phi_{0}(-x, y), & x \rightarrow-\infty \\
T \phi_{0}(x, y), & x \rightarrow+\infty\end{cases}
\end{aligned}
$$

where $\nabla^{2}$ is the two-dimensional Laplacian operator.

It may be pointed out, at this stage, that unique solution of the above BVP will exist for all smooth functions $c(x)$ which are of compact support, as can be easily established by the use of standard ideas for BVPs, involving elliptic partial differential equations.

In view of the boundary condition (12) and the fact that a wave train propagating in uniform finite depth experiences no reflection, we can express $\phi, R$ and $T$ in terms of the perturbation parameter $\varepsilon$ as

$$
\begin{gathered}
\phi=\phi_{0}+\varepsilon \phi_{1}+O\left(\varepsilon^{2}\right), \quad R=\varepsilon R_{1}+O\left(\varepsilon^{2}\right), \\
T=1+\varepsilon T_{1}+O\left(\varepsilon^{2}\right) .
\end{gathered}
$$

It must be noted that such a perturbation expansion ceases to be valid at Bragg resonance when the reflection coefficient becomes much larger than the undulation parameter, as pointed out by Mei [8].

Applying the expansions (14) in Eqs. (10)-(13), we find after equating the coefficients of $\varepsilon$ from both sides that we can formulate a BVP for the function $\phi_{1}(x, y)$ which satisfies the Eq. (10) and the condition (11) together with the following other conditions:

$$
\begin{gathered}
\frac{\partial \phi_{1}}{\partial y}-G^{\prime} \phi_{1}=\mathrm{i} \mu \frac{\mathrm{d}}{\mathrm{d} x}\left\{c(x) \mathrm{e}^{\mathrm{i} \mu x}\right\}-v^{2} c(x) \mathrm{e}^{\mathrm{i} \mu x} \\
+G^{\prime} k_{0} A c(x) \mathrm{e}^{\mathrm{i} \mu x} \equiv V(x)(\text { say }), \quad \text { on } y=h
\end{gathered}
$$

and

$\phi_{1}(x, y) \sim \begin{cases}R_{1} \phi_{0}(-x, y), & \text { as } x \rightarrow-\infty \\ T_{1} \phi_{0}(x, y), & \text { as } x \rightarrow+\infty\end{cases}$

where $A=\left(K \cosh k_{0} h-k_{0} \sinh k_{0} h\right) /\left(K \sinh k_{0} h-\right.$ $\left.k_{0} \cosh k_{0} h\right)$.

By using the Fourier transform technique, we easily find the solution of the above BVP for $\phi_{1}$, given by 
$\phi_{1}(x, y)=\lim _{\mu^{\prime} \rightarrow 0} \frac{1}{2 \pi} \int_{-\infty}^{\infty} \frac{F(\widehat{\xi}, y)}{G_{\mu^{\prime}}(\widehat{\xi}, h)} \mathrm{e}^{-\mathrm{i} \xi x} \mathrm{~d} \xi$,

where

$F(\widehat{\xi}, y)=[\widehat{\xi} \cosh \widehat{\xi} y-\widehat{K} \sinh \widehat{\xi} y] \bar{V}(\xi)$,

$G_{\mu^{\prime}}(\widehat{\xi}, h)=\widehat{\xi}\left[\left(\widehat{\xi}+\widehat{K} G^{\prime} / \widehat{\xi}\right) \sinh \widehat{\xi} h-\left(\widehat{K}+G^{\prime}\right) \cosh \widehat{\xi} h\right]$,

$\widehat{\xi}^{2}=\xi^{2}+v^{2}, \quad \bar{V}(\xi)=\int_{-\infty}^{\infty} V(x) \mathrm{e}^{\mathrm{i} \xi x} \mathrm{~d} x$

We note that the Fourier transform, used above, exists if only we make an artificial assumption that $K$ possesses a small imaginary part so that $K$ is replaced by $\widehat{K}=\left(\sigma^{2}+\mathrm{i} \mu^{\prime} \sigma\right) / g \equiv$ $K+\mathrm{i} \mu^{\prime} \sigma / g$, where $\mu^{\prime}>0$ is very small which has been taken to tend to zero in the above result (16).

All the important results can then be derived by applying contour integration method to the relation (16).

We observe that the integral in (16) has certain singularities at the zeros of the expression (18). If $\widehat{K}=\widehat{K}_{1}+\mathrm{i} \widehat{K}_{2}$, and if $\hat{\zeta}=\alpha+\mathrm{i} \beta$ is a zero of (18), then $\hat{\zeta}$ can be determined as

$\hat{\zeta}= \pm \alpha_{n} \pm \mathrm{i} \beta_{n}, \quad$ and $\quad \hat{\zeta}= \pm\left(k_{0}+\gamma\right) \pm \mathrm{i} \beta_{n}^{\prime}$,

where $\alpha_{n}, \gamma$ and $\beta_{n}^{\prime}$, all are of $O\left(\widehat{K}_{2}\right)$ and $\beta_{n}$ satisfies the transcendental equation

$\left(\beta-\widehat{K}_{1} G^{\prime} / \beta\right) \tan \beta h+\left(\widehat{K}_{1}+G^{\prime}\right)=0$.

Thus, in the limit, when $\mu^{\prime} \rightarrow 0$ (also $\widehat{K_{2}} \rightarrow 0$ ), we find that $\alpha_{n} \rightarrow 0, \gamma \rightarrow 0$ and $\beta_{n}^{\prime} \rightarrow 0$, so that the reflected and transmitted wave terms in the expression for $\phi_{1}$ as given by the expression (16) can be determined by utilizing the effect of the poles at $\hat{\zeta}= \pm\left(k_{0}+\gamma\right) \pm \mathrm{i} \beta_{n}^{\prime}$.

Hence, we find that the reflection and transmission coefficients $R_{1}$ and $T_{1}$ are obtainable in the following forms:

$$
\begin{aligned}
R_{1}= & \frac{-2 \mathrm{i} B \sec \theta\left(k_{0}^{2} \cos 2 \theta+G^{\prime} k_{0} A\right)}{2\left(k_{0}+G^{\prime} K / k_{0}\right) h+\left(1-G^{\prime} K / k_{0}^{2}\right) \sinh 2 k_{0} h} \\
& \times \int_{-\infty}^{\infty} c(x) \mathrm{e}^{2 \mathrm{i} \mu x} \mathrm{~d} x
\end{aligned}
$$

and

$$
\begin{aligned}
T_{1}= & \frac{-2 \mathrm{i} B \sec \theta\left(-k_{0}^{2}+G^{\prime} k_{0} A\right)}{2\left(k_{0}+G^{\prime} K / k_{0}\right) h+\left(1-G^{\prime} K / k_{0}^{2}\right) \sinh 2 k_{0} h} \\
& \times \int_{-\infty}^{\infty} c(x) \mathrm{d} x .
\end{aligned}
$$

For the special case with negligible porosity of the sea-bed, i.e., when $G^{\prime}=0$, the results (21) and (22) agree with the ones obtained by Miles [9], Mandal and Basu [5] (for the case of absence of surface tension) and Martha and Bora [6].

Numerical results for the reflection and transmission coefficients as given by the relations (21) and (22) can be obtained once the shape function $c(x)$ is known.

\section{A special bed surface}

As a particular example we consider the undulation function $c(x)$ to represent sinusoidal ripples so that $c(x)$ is given by

$c(x)= \begin{cases}a \sin (l x), & \frac{-m \pi}{l} \leq x \leq \frac{m \pi}{l} \\ 0 & \text { otherwise, }\end{cases}$

where $a$ is the ripple amplitude, $l$ the ripples wave number and $m$ a positive integer. The basic reason in taking up such a sinusoidal representation of the sea-bed is that any general sufficiently smooth but undulated form of the sea-bed can be represented in the form of a Fourier sine series and then, each term of the resulting Fourier series can be handled with the aid of the types of results derived here.

We find in this special case, that

$$
\begin{aligned}
R_{1}= & \frac{4 B \sec \theta\left(k_{0}^{2} \cos 2 \theta+G^{\prime} k_{0} A\right)}{2\left(k_{0}+G^{\prime} K / k_{0}\right) h+\left(1-G^{\prime} K / k_{0}^{2}\right) \sinh 2 k_{0} h} \\
& \times \frac{(-1)^{m+1} a l}{l^{2}-(2 \mu)^{2}} \sin \left(\frac{2 \mu m \pi}{l}\right)
\end{aligned}
$$

and

$T_{1}=0$.

These results match exactly with those obtained by Davies and Heathershaw [3] when $G^{\prime}=0$ and $\theta=0$. Eq. (24) illustrates that for a given number of $m$ ripples, the first order wave reflection coefficient is an oscillatory function. Furthermore, if the bed wave number is twice the component of the wave number along $x$-axis $(2 \mu=l)$, then Eq. (24) reveals that there is a Bragg resonance between the surface waves and bed forms as described by Davies [1,2] and Mei [8] and reported by Heathershaw [4]. Hence, at resonance $(2 \mu=l)$, we obtain

$$
R_{1}=\frac{2 B \sec \theta\left(k_{0}^{2} \cos 2 \theta+G^{\prime} k_{0} A\right)}{2\left(k_{0}+G^{\prime} K / k_{0}\right) h+\left(1-G^{\prime} K / k_{0}^{2}\right) \sinh 2 k_{0} h} \frac{a m \pi}{l},
$$

which shows that $R_{1}$ becomes a constant multiple of $m$, the number of ripples in the patch. It indicates that relatively few bottom undulations with its wave number equal to approximately twice the $x$-component of the surface wave number, may give rise to a very substantial reflected wave. A possible consequence of this is a coupling between ripple growth and wave reflection, which may be important in the problems of coastal protection.

\section{Numerical results}

The numerical computation is presented here for the first order reflection coefficient as given by Eq. (24). From the Figs. 1 and 2 which are oscillatory in nature, it is clear that peak values of $\left|R_{1}\right|$ are attained when the wave number of the bottom undulations $l h$ becomes approximately twice the surface wave number $k_{0} h$. Also, at Bragg resonance the value of the reflection coefficient increases as the porous effect parameter increases. 


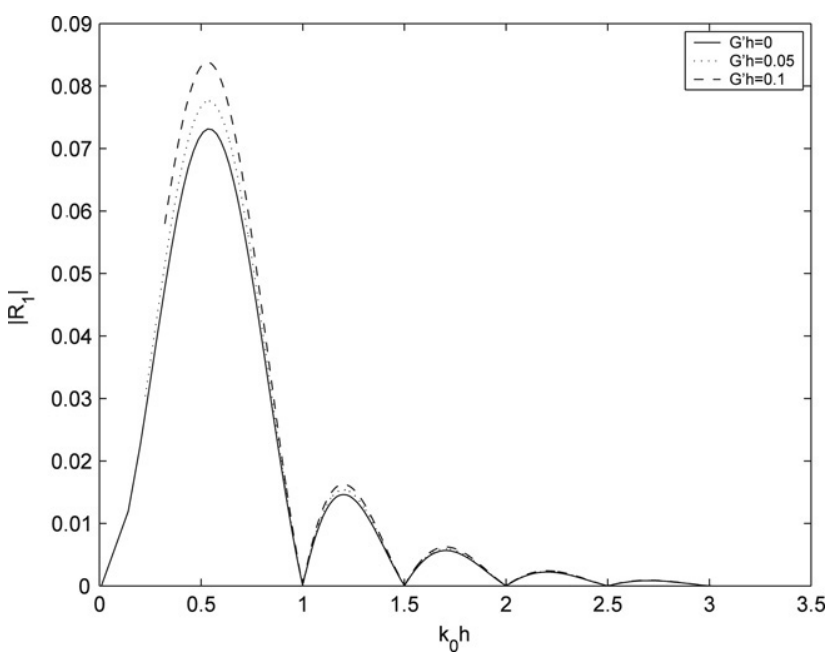

Fig. 1. Reflection coefficient against the wave number $k_{0} h$ for $\theta=0$; $a / h=0.1 ; l h=1 ; m=1$.

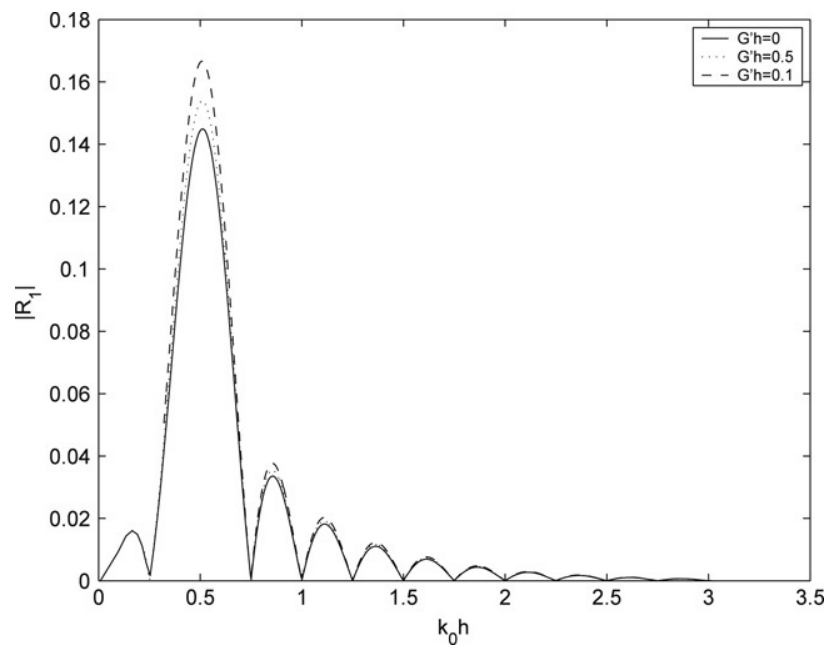

Fig. 2. Reflection coefficient against the wave number $k_{0} h$ for $\theta=0 ; a / h=$ $0.1 ; l h=1 ; m=2$.

In contrast to Fig. 1, which is for a single ripple only, we find in Fig. 2, which is for double ripples, that the overall value of $\left|R_{1}\right|$ is increased and the oscillating nature of $\left|R_{1}\right|$ against $k_{0} h$ is more pronounced. Also, like in the single ripple case, at Bragg resonance the value of the reflection coefficient increases as the porous effect parameter increases.

The effects of variation of $\theta$ are shown in the graphs represented in Figs. 3 and 4. It is clear that for $G^{\prime} h=0$, $\left|R_{1}\right|$ vanishes independently of the shape of the function at $\theta=\frac{\pi}{4}$ which validates Eq. (21). From the graphs it is also found that even for very small values of $G^{\prime} h,\left|R_{1}\right|$ does not vanish independently of the shape of the function at $\theta=\frac{\pi}{4}$.

\section{Conclusion}

Fourier transform method is used to solve the problem of water-wave scattering by small undulation on a porous sea-bed for oblique incidence. The results obtained here agree with the known ones, obtained earlier, in the case when the bed has no porous effect. From the computational results it is observed

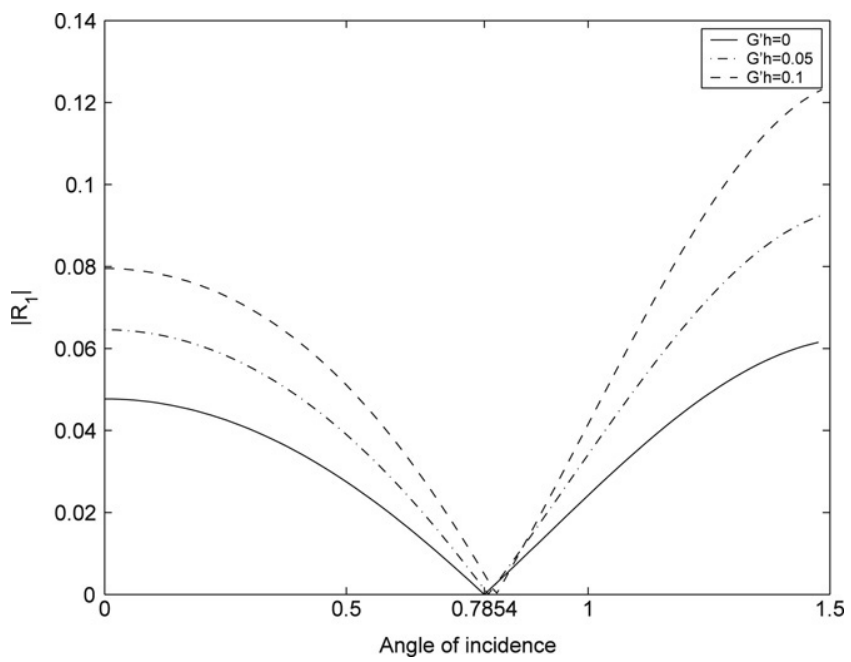

Fig. 3. Reflection coefficient against the angle of incidence $\theta$ for $K h=0.1$; $a / h=0.1 ; l h=1 ; m=1$.

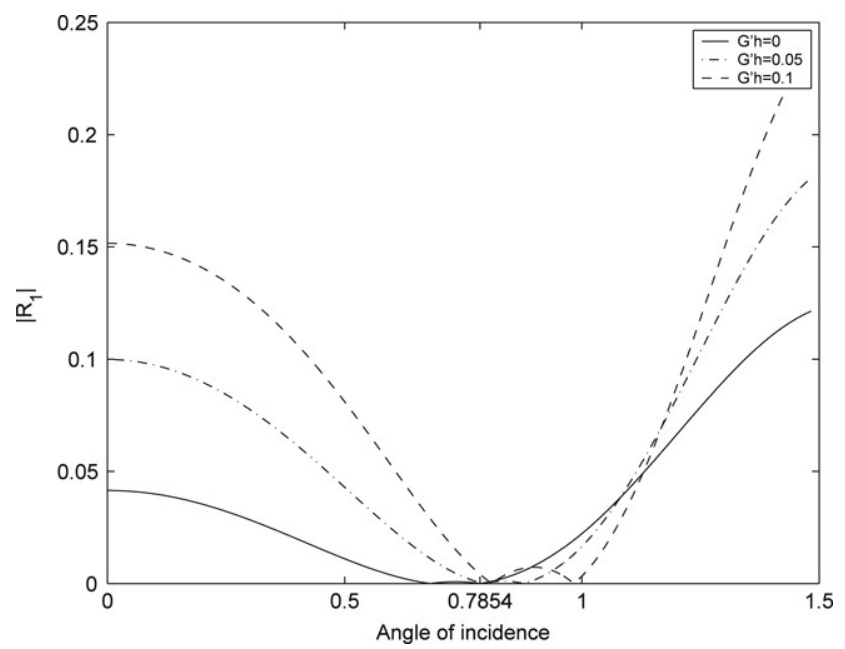

Fig. 4. Reflection coefficient against the angle of incidence $\theta$ for $K h=0.1$; $a / h=0.1 ; l h=1 ; m=2$.

that the reflection coefficient increases with increasing porous effect.

\section{Acknowledgement}

The first author is grateful to the Council of Scientific and Industrial Research (CSIR), Government of India for providing senior research fellowship.

\section{References}

[1] Davies AG. On the interaction between surface waves and undulations on the sea bed. J Marine Res 1982;40:331-68.

[2] Davies AG. The reflection of wave energy by undulations of the sea bed. Dyn Atms Oceans 1982;6:207-32.

[3] Davies AG, Heathershaw AD. Surface-wave propagation over sinusoidally varying topography. J Fluid Mech 1984;144:419-43.

[4] Heathershaw AD. Seabed-wave resonance and sand bar growth. Nature 1982;296:343-5.

[5] Mandal BN, Basu U. A note on oblique water-wave diffraction by a cylindrical deformation of the bottom in the presence of surface tension. Arch Mech 1990;42(6):723-7. 
[6] Martha SC, Bora SN. Water wave diffraction by a small deformation of the ocean bottom for oblique incidence. Acta Mech 2006;185(3-4):165-77.

[7] Mase H, Takeba K. Bragg scattering of gravity waves over a porous ripple bed. In: Proc. 24th ICCE. Kobe (Japan): ASCE; 1994. p. 635-49.

[8] Mei CC. Resonant reflection of surface water waves by periodic sand-bars. J Fluid Mech 1985;152:315-35.
[9] Miles JM. Oblique surface wave diffraction by a cylindrical obstacle. Dyn Atms Oceans 1981;6:121-3

[10] Silva R, Salles P, Palacio A. Linear wave propagating over a rapidly varying finite porous bed. Coastal Eng 2002;44:239-60.

[11] Zhu S. Water waves within a porous medium on andulating bed. Coastal Eng 2001;42:87-101. 